\title{
Violencia de género: reflexiones conceptuales, derivaciones prácticas
}

\author{
Eva Espinar Ruiz \\ Miguel Ángel Mateo Pérez \\ Universidad de Alicante. \\ Departamento de Sociología II \\ Campus de S. Vicente del Raspeig. Ap. 99. 03080 Alicante \\ Eva.Espinar@ua.es \\ MA.Mateo@ua.es
}

\section{Resumen}

El objetivo de este artículo es analizar el término violencia de género, destacando sus características y potencialidades, tanto teóricas como prácticas. En este sentido, puede definirse la violencia de género como aquella violencia basada en las relaciones y definiciones de género dominantes en una sociedad dada. Partiendo de esta definición, el centro de atención ha de situarse en los fundamentos socioculturales de esta violencia y en las posibles transformaciones que en este ámbito puedan tener lugar.

Palabras clave: género, violencia, violencia estructural, violencia cultural.

\section{Abstract. Gender violence: concepts and practical derivations}

In this article we analyse the term gender violence, pointing out its theoretical and practical characteristics and potentialities. In this sence, gender violence can be defined as that violence based on prevalent gender relations and definitions. Hence, the center of atention must concentrate on the sociocultural bases of this violence and in their transformation.

Key words: gender, violence, structural violence, cultural violence.

\section{Sumario}

1. Introducción 4. Efectos de la participación

2. Fundamentos conceptuales: violencia y género

3. Violencia de género: violencia directa, estructural y cultural de las mujeres en el mercado laboral en términos de violencia cultural y directa

5. Conclusiones

Bibliografía 


\section{Introducción}

El término violencia de género se ha convertido en una expresión de uso frecuente, y no sólo en ámbitos feministas y/o académicos, sino también en los medios de comunicación, en el lenguaje institucional o incluso en las conversaciones cotidianas. Ahora bien, ¿qué es, exactamente, la violencia de género? ¿Qué rasgos diferencian la violencia de género de otras formas de violencia? ¿Resulta analíticamente útil emplear este término? A estas y otras preguntas semejantes trataremos de dar respuesta a lo largo del presente artículo.

En definitiva, pretendemos justificar la elección del término violencia de género frente al resto de los empleados en este campo de estudio: violencia contra las mujeres, violencia doméstica, terrorismo patriarcal, malos tratos, etc. Términos que, incluido el de violencia de género, hacen referencia a diferentes dimensiones y responden a distintos planteamientos teóricos e, incluso, políticos.

Ciertamente, en este debate terminológico, el uso del término violencia de género no goza de pleno consenso e incluso llega a ser rechazado por algunas destacadas representantes del movimiento feminista (Rivera, 2001). Entre otras cuestiones, estas autoras plantean que hablar de violencia de género supone emplear una categoría neutra que oculta la dominación masculina, es decir, oculta quién es la víctima y quién el agresor. Así, defienden el empleo de términos como el de violencia contra las mujeres o el de violencia de los hombres contra las mujeres (Tubert, 2003: 8).

Por otra parte, desde posturas críticas a los planteamientos teóricos feministas, se cuestiona, directamente, la capacidad explicativa de la variable género a la hora de estudiar la violencia, situando el centro de atención en otras variables. Estas teorías están dando lugar a un elevado número de publicaciones a lo largo de los últimos años (Follingstad y otros, 2002; HoltzworhtMunroe y otros, 1997; Felson, 2002; Dutton y Kropp, 2000).

Frente a tales planteamientos, como se ha indicado anteriormente, el objeto de reflexión de este artículo es el propio concepto de violencia de género, cuyo empleo consideramos fundamental para la comprensión de determinadas formas de violencia.

En cuanto a su estructura interna, el presente artículo se inicia con una breve introducción conceptual en torno a los términos violencia y género, para proceder, a continuación, a su análisis conjunto y, por tanto, al concepto de violencia de género. De esta forma, analizaremos los fundamentos estructurales y culturales de esta violencia, es decir, los pilares que la sostienen y que, en sí mismos, pueden considerarse formas de violencia. Finalmente, nos centraremos en el estudio de las posibles relaciones existentes entre cambios estructurales y culturales y sus efectos sobre la violencia directa, tratando de establecer algunas derivaciones prácticas de estas relaciones. Cerraremos el artículo con un resumen de las principales conclusiones y la bibliografía empleada. 


\section{Fundamentos conceptuales: violencia y género}

A pesar de la bibliografía existente, se carece de un acuerdo, mínimamente amplio, en torno a la definición más adecuada de violencia, de forma que se viene optando por diferentes definiciones según disciplinas, presupuestos teóricos de partida u objetivos de investigación.

La definición más restringida de violencia supone identificarla con actos de violencia, fundamentalmente física, entre personas concretas. Esta violencia física directa ha protagonizado gran parte de las investigaciones desarrolladas sobre la violencia en general y contra las mujeres en particular, puesto que es la forma de violencia que ofrece más facilidades en cuanto a su tratamiento empírico.

Sin embargo, una aproximación de este tipo puede llevar a identificar la violencia con actos aislados, sin tener en cuenta su posible reiteración en el tiempo y su complementariedad con otros comportamientos. Igualmente, estaría excluyendo el análisis de formas de violencia, de naturaleza no estrictamente física, pero cuya gravedad es recurrentemente destacada por las propias víctimas. Éste es el caso de las diversas formas de violencia psicológica, que, en palabras de Marie-France Irigoyen (1998), pueden llegar a «hacer pedazos» a cualquier persona.

Ahora bien, aún atendiendo a formas de violencia no estrictamente físicas, autores como Johan Galtung (cuyos conceptos aplicaremos en este artículo) continúan considerando limitado el análisis. Galtung emplea el término violencia directa para referirse a aquella violencia física y/o verbal fácilmente visible en forma de conductas, y plantea la necesidad de atender a otras dos formas de violencia, de más difícil identificación: la violencia estructural y la cultural.

La violencia estructural hace referencia a situaciones de explotación, discriminación, marginación o dominación (Tortosa, 1992: 137). Se trata de una violencia «edificada dentro de la estructura y [que] se manifiesta como un poder desigual y, consiguientemente, como oportunidades de vida distintas. Los recursos están desigualmente distribuidos y [...] por encima de todo, está desigualmente repartido el poder de decisión acerca de la distribución» de los mismos. En este sentido, «la fórmula general que está detrás de la violencia estructural es la desigualdad» y la injusticia social (Galtung, 1995). Por su parte, son violencia cultural todos aquellos razonamientos, actitudes e ideas que justifican, legitiman y promueven la violencia en sus formas directa o estructural (Tortosa, 2002: 27). Así, la cultura puede llevar a ver la explotación y/o la represión como normales y naturales, o simplemente a no llegar a verlas como tales (Galtung, 1990: 295).

Galtung justifica el hecho de adoptar una definición tan amplia de violencia al considerar que «la violencia está presente cuando los seres humanos se ven influidos de tal manera que sus realizaciones efectivas, somáticas y mentales están por debajo de sus realizaciones potenciales», de modo que "cuando lo potencial es mayor que lo efectivo, y ello es evitable, existe violencia» (1995: 314-315). 
Partiendo de este esquema, sería necesario atender a las tres dimensiones de la violencia para analizar gran parte de los fenómenos violentos. Así, Galtung analiza cómo estas formas de violencia están íntimamente relacionadas entre sí, conduciendo unas a otras (violencia estructural a directa, cultural a estructural, etc.). La paz, como reverso del triángulo de la violencia, no puede reducirse a la mera ausencia de violencia directa, sino que implica la actuación sobre los tres vértices del triángulo.

Antes de aplicar este esquema al caso concreto de la violencia de género, necesitamos definir el segundo componente de este binomio: el concepto de género (Sau, 2000: 133-138; Cobo, 1995).

Aunque el término género debe su sistematización al resurgir de la reflexión feminista, fundamentalmente anglosajona, en los años sesenta y setenta del siglo XX, representa la recuperación de planteamientos ya implícitos en las obras de autores anteriores como Poulain de la Barre (siglo XVII), Condorcet, Olympe de Gouges o Mary Wollstonecraft (siglo XVIII).

Aún sin emplear, explícitamente, el término género, podemos encontrar su definición en El segundo sexo de Simone de Beauvoir (1949), donde la autora afirma que "una mujer no nace, sino que se hace», es decir que aquellas características de las mujeres que podríamos denominar "femeninas» no son fruto de la naturaleza, sino que son aprendidas a través de un complejo proceso individual y social. Es, precisamente, ese carácter sociocultural de las diferencias entre hombres y mujeres lo que pretende recoger el término género.

Hablar de género supone distinguirlo del término sexo. Sexo se emplea para hacer referencia a la base biológica de las diferencias entre hombres y mujeres, mientras que género hace referencia a la construcción sociocultural sobre la base biológica, es decir, a los roles, funciones, comportamientos, actitudes, identidades, etc. que las sociedades adjudican a cada sexo y que los seres humanos aprenden e interiorizan. Como plantea Inés Alberdi, estas diferencias «se marcan muchas veces de forma tan indeleble que no es raro que mucha gente, incluso hoy día, haya pensado que se trata de rasgos biológicos y naturales» (1999: 16).

El reconocimiento de la naturaleza sociocultural de las diferencias (y desigualdades) de género tiene una consecuencia directa en términos prácticos: cabe la posibilidad de modificarlas. En este sentido, el concepto de género es fiel reflejo de la doble naturaleza teórica y política del feminismo, puesto que uno de los objetivos de sus sistematizadoras era denunciar que la situación social de las mujeres obedecía a una construcción social y no a un destino natural o biológico, y que tal situación podía modificarse (Reverter, 2003: 39).

Dicho esto, y a diferencia de lo que muchas veces parece interpretarse, "género hace referencia al género masculino y al género femenino" (López y Sierra, 2001: 4). Género no es sinónimo de mujer, puesto que los hombres también tienen género. De hecho, desde los años ochenta, recibe una creciente atención el estudio de la construcción social de las masculinidades, especialmente en relación con el análisis de la violencia de género (Miedzian, 1991; Breines y otros, 2000; Pringle y otros, 2001; Brod y Kaufman, 1994; Corsi y Bonino, 2003). 
Esta explicación, aunque útil, empieza a ser calificada como simplista por autoras que apuestan por cuestionar la estricta distinción entre sexo y género. Se trata de planteamientos que optan por incluir en el análisis factores socioculturales y biológicos, así como las mutuas interacciones entre ambos (Connell, 1995; Butler, 1990). Aún así, estos razonamientos no llegan a anular la capacidad analítica del concepto de género; término fundamental para entender identidades, relaciones y determinadas formas de violencia, como veremos a continuación.

\section{Violencia de género: violencia directa, estructural y cultural}

El término violencia de género hace referencia a aquellas formas de violencia que hunden sus raíces en las definiciones y relaciones de género dominantes en una sociedad dada. Partiendo de esta definición, cabe hacer una doble aclaración. Por una parte, no todas las formas de violencia contra las mujeres pueden calificarse, propiamente, de violencia de género. Es decir, para algunas formas de violencia, las explicaciones pueden situarse en variables distintas a las relacionadas con el género (Izquierdo, 1998). Por otra parte, también podemos aplicar una perspectiva de género al análisis de otras formas de violencia, no únicamente aquéllas que tienen por víctimas a mujeres. Por ejemplo, Michael Kaufman utiliza el término tríada de la violencia para referirse a la estrecha relación existente entre la violencia de los hombres contra las mujeres, la violencia de los hombres contra otros hombres y la internalización de la violencia, es decir, la violencia contra uno mismo. Esta tríada de la violencia estaría ligada a la construcción e interiorización de las masculinidades dominantes en la mayor parte de las sociedades conocidas (Kaufman, 1999: 66).

Igualmente, de acuerdo con las definiciones de violencia expuestas en el epígrafe anterior, al hablar de violencia de género no nos estamos refiriendo, exclusivamente, a actos claros de violencia directa (física, psicológica, sexual, económica o social), sino también a los más complejos de violencia estructural y cultural ${ }^{1}$. Así, tanto las estructuras patriarcales como la ideología machista son, en sí mismas, formas de violencia basadas en el género, a la vez que fundamentan, explican y justifican las distintas manifestaciones de violencia directa.

Estas tres formas de violencia (directa, estructural y cultural) mantendrían, entre sí, una estrecha interrelación, tal y como plantea Johan Galtung. La violencia estructural puede desembocar en violencia directa; la cultural, en estructural y así sucesivamente. Sin embargo, de acuerdo con las palabras de Carmen Magallón, podemos afirmar que de todas estas interrelaciones, el flujo principal sería «el que va de la violencia cultural a la violencia directa pasan-

1. Para una aplicación de esta terminología acuñada por Johan Galtung a la violencia de género, puede consultarse: Galtung, 1996: 40-48; Magallón, 2005; Martínez, 2005, y Torres, 2001. 
do por la estructural» (2005: 37), como trataremos de mostrar en el siguiente epígrafe ${ }^{2}$.

De esta definición se deduce que, si el objetivo es poner fin a la violencia de género, las actuaciones han de ir dirigidas a sus diferentes componentes. Es decir, no es suficiente, aunque irrenunciable, la actuación sobre la violencia directa (defensa, protección y recuperación de las víctimas, así como castigo a los agresores), sino que es necesario actuar también sobre las otras dos formas de violencia: la violencia estructural y la cultural.

Siguiendo estos planteamientos, y con el fin de analizar los posibles efectos de algunas transformaciones estructurales y culturales sobre la violencia directa, nos centraremos, en las próximas páginas, en el estudio de los cambios derivados de la masiva incorporación de las mujeres al mercado laboral que ha tenido lugar en las últimas décadas en los países desarrollados, con notables diferencias entre países en cuanto a ritmos y niveles.

\section{Efectos de la participación de las mujeres en el mercado laboral en términos de violencia cultural y directa}

En primer lugar, podemos afirmar que la propia incorporación (o, hablando en términos históricamente más apropiados, reincorporación) de las mujeres al mercado laboral es interpretable, en sí misma, como una reducción de la violencia estructural. Cuestiones como el aumento paulatino de las tasas de actividad $^{3}$ o la presencia de mujeres en una mayor variedad de esferas laborales pueden interpretarse como un indicador del retroceso de la discriminación social históricamente vivida por las mujeres.

Ahora bien, en qué medida podemos hablar de cambios en las relaciones de poder en el interior de las familias o de cambios en términos de roles, de identidades o en términos de violencia directa, derivados de esta participación laboral. Sin dejar de reconocer la importancia histórica de esta participación, en las próximas líneas nos centraremos en el análisis de sus efectos en términos de violencia cultural y directa.

Podemos comenzar analizando las características de este aumento generalizado en las tasas de actividad femenina ${ }^{4}$. Así, y hablando siempre en términos generales, puede afirmarse que la reincorporación al mercado laboral no está suponiendo un abandono de las responsabilidades femeninas tradicionales en el interior del espacio doméstico ${ }^{5}$. En palabras de Gilles Lipovetsky, en todo

2. Pueden consultarse los siguientes estudios sobre el papel de la violencia cultural en términos de género a la hora de justificar y respaldar la violencia directa: Nayak y otros, 2003; Campbell, 1993; Gabler y otros, 1998; y Markowitz, 2001.

3. Para un análisis crítico de los indicadores laborales clásicos, como el de tasa de actividad, pueden consultarse: Carrasco y Mayordomo, 2000; Prieto, 1999, y Carrasco y otros, 2004.

4. Para el caso español, pueden consultarse los estudios de Maruani, 2004, y Castaño, 2004.

5. Pueden consultarse los diferentes estudios existentes sobre los usos del tiempo de hombres y mujeres; por ejemplo: Raldúa, 2001. 
caso, «lo que ha cambiado no es tanto la lógica de la división sexual de los roles familiares como el surgimiento de una mayor cooperación masculina en el seno del ámbito tradicional, basado en la preponderancia femenina» (1997: 231). De esta forma, la participación laboral de las mujeres no parece estar teniendo, como consecuencia, un cuestionamiento profundo de los roles tradicionales, por lo menos por lo que respecta al interior del núcleo familiar.

Por otra parte, uno de los rasgos característicos de la participación laboral de las mujeres es su segmentación horizontal. Este término hace referencia a la presencia desproporcionada de mujeres en un relativamente reducido número de ocupaciones, lo cual da lugar a sectores laborales feminizados. Estos sectores económicos suelen estar relacionados con roles y funciones que históricamente le han sido asignados a las mujeres, y, por tanto, supone externalizar al mercado laboral la tradicional división sexual del trabajo. Esta característica de la participación laboral de las mujeres parece que se mantendrá, como mínimo, en un futuro inmediato, si atendemos a las carreras educativas que actualmente están siguiendo chicos y chicas, tanto en el nivel universitario como en el no universitario. Una vez más, podemos plantearnos serias dudas en cuanto a las posibilidades que esta participación laboral representa en cuanto al cuestionamiento de roles e identidades.

Igualmente, las mujeres tienen mayores probabilidades de mantener relaciones discontinuas y precarias con el mundo laboral, lo que dificulta sus posibilidades de independencia y autonomía. Las mujeres, en casi todos los países desarrollados, presentan mayores tasas de paro que los hombres y tienen el protagonismo en los contratos a tiempo parcial, temporales y en la economía sumergida. De alguna manera, socialmente se sigue considerando más tolerable el desempleo femenino que el masculino, así como la mayor precariedad laboral que afecta al colectivo de mujeres.

En conclusión, podemos afirmar que el aumento generalizado de las tasas de actividad se ve acompañado por una clara discriminación de las mujeres en el ámbito laboral, que se refleja en menos derechos laborales, salarios más bajos, menor prestigio social, derechos reducidos a prestaciones económicas (López y Valiño, 2003), etc. De esta forma, persiste una violencia estructural a pesar de los cambios, con lo cual disminuyen sus posibles efectos, tanto culturales como en términos de violencia directa.

Por otra parte, esta violencia estructural se justifica a partir de la permanencia de una serie de creencias sobre la mujer y su capacidad productiva, sobre sus roles, aptitudes e identidades (violencia cultural a fin de cuentas), que se adaptan a las necesidades del modelo económico imperante. En este sentido, Margaret Maruani afirma que «de hecho, existen pocos ámbitos donde una mutación social de una amplitud tal se haya llevado a cabo sobre un fondo de desigualdades tan obstinadas como rebeldes» (2004: 61).

Desde otro nivel de análisis, y empleando datos de encuesta (aún con las limitaciones de este tipo de datos en el estudio de la violencia de género), podemos, directamente, analizar la posible relación entre participación laboral de las mujeres y violencia directa, en este caso al interior del núcleo familiar. Para 
ello, nos centraremos geográficamente en España y emplearemos datos de una encuesta elaborada y aplicada desde la Universidad de Alicante, en el año 2001. Los autores de este artículo formaban parte del equipo de investigadores que la diseñaron y analizaron ${ }^{6}$.

Respecto a las preguntas dirigidas a la detección de casos de violencia, esta encuesta se inspira en las aplicadas por el Instituto de la Mujer en sus macroencuestas sobre la violencia contra las mujeres. A través de estas preguntas, se intenta detectar dos tipos de violencia: la de tipo A y la de tipo B. La violencia de tipo A hace referencia a aquellas mujeres que el investigador califica como víctimas de violencia a partir de sus respuestas a una serie de preguntas, independientemente de cómo se califiquen a sí mismas. El tipo de violencia $\mathrm{B}$ hace referencia a aquellas mujeres que, expresamente, se autocalifican como maltratadas.

Tanto en nuestro estudio como en el del Instituto de la Mujer, los datos no permiten concluir, de ninguna de las maneras, que la participación laboral femenina proteja a las mujeres de situaciones violentas dentro del hogar. En todo caso, podría hablarse de una relación, si bien apenas significativa, en el sentido contrario (resultados semejantes obtiene Meil, 2004).

En la tabla 1, presentamos los resultados obtenidos al analizar la posible relación entre autocalificarse como víctima de violencia (violencia tipo B) y la situación laboral de la mujer encuestada, agrupada ésta última en tres categorías.

En esta tabla, los índices estadísticos asociados ${ }^{7}$ señalan la ausencia de relación significativa entre ambas variables. Es decir, estadísticamente, no parece haber relación entre actividad laboral y sentirse víctima de violencia (violencia de tipo B). Ahora bien, si analizamos en mayor detalle la tabla 1 y nos fijamos en los residuos corregidos ${ }^{8}$, podemos hablar de una mayor probabilidad de padecer violencia de tipo B si la mujer trabaja o está jubilada (residuo corregido superior a 1,9$)$.

En la tabla 2, realizamos el mismo ejercicio para el caso de las mujeres que, objetivamente, puede considerarse que se encuentran en situaciones de violencia (violencia de tipo A).

6. Encuesta realizada dentro del proyecto de investigación: Indicadores dinámicos para el estudio del empobrecimiento de las mujeres; financiado por el Instituto de la Mujer y dirigido por el profesor José María Tortosa. Pueden consultarse: Tortosa, 2001 y Tortosa, 2002. El universo de esta encuesta es la población de sexo femenino de 18 y más años residente en el territorio español peninsular; con una muestra de 1.200 casos. Para un nivel de confianza del $95,5 \%$ (dos sigmas), y $P=Q$, el error es de $\pm 2,5$ para el conjunto de la muestra.

7. Un Chi-cuadrado de Pearson de 5,218; 2 grados de libertad y 0,074 de significación asintótica.

8. Los residuos corregidos se calculan a partir de las diferencias entre las frecuencias observadas y las que habríamos obtenido en el caso de que no hubiera relación entre las dos variables (esperadas), pero se corrigen por el tamaño de las frecuencias de cada categoría y por la varianza de las celdas. La utilidad de estos residuos está en que tienen una distribución aproximadamente normal con una media igual a 0 y desviación típica igual a 1 . De tal forma que un valor del residuo corregido mayor que $\pm 1,96$ se interpreta como una desviación significativa de las frecuencias esperadas para esa celda. 
Tabla 1. Tabla de contingencia entre situación laboral y violencia tipo B.

\begin{tabular}{|c|c|c|c|c|c|c|}
\hline & & & \multicolumn{3}{|c|}{ Situación laboral } & \multirow[b]{2}{*}{ Total } \\
\hline & & & $\begin{array}{l}\text { Trabaja } \\
\text { o jubilada }\end{array}$ & $\begin{array}{l}\text { Pensionista, } \\
\text { parada, } \\
\text { estudiante... }\end{array}$ & $\begin{array}{l}\text { Trabajo } \\
\text { doméstico }\end{array}$ & \\
\hline \multirow[t]{4}{*}{$\begin{array}{l}\text { Violencia } \\
\text { tipo B }\end{array}$} & Sí & \% situación laboral & $8,0 \%$ & $5,8 \%$ & $4,1 \%$ & $6,3 \%$ \\
\hline & & Residuos corregidos & 2,0 & $-0,5$ & $-1,8$ & \\
\hline & No & \% situación laboral & $92,0 \%$ & $94,2 \%$ & $95,9 \%$ & $93,7 \%$ \\
\hline & & Residuos corregidos & $-2,0$ & 0,5 & 1,8 & \\
\hline Total & & \% situación laboral & $100 \%$ & $100 \%$ & $100 \%$ & $100 \%$ \\
\hline
\end{tabular}

Tabla 2. Tabla de contingencia entre situación laboral y violencia tipo A.

\begin{tabular}{|c|c|c|c|c|c|c|}
\hline & & & \multicolumn{3}{|c|}{ Situación laboral } & \multirow[b]{2}{*}{ Total } \\
\hline & & & $\begin{array}{l}\text { Trabaja } \\
\text { o jubilada }\end{array}$ & $\begin{array}{l}\text { Pensionista, } \\
\text { parada, } \\
\text { estudiante... }\end{array}$ & $\begin{array}{l}\text { Trabajo } \\
\text { doméstico }\end{array}$ & \\
\hline \multirow[t]{4}{*}{$\begin{array}{l}\text { Violencia } \\
\text { tipo A }\end{array}$} & \multirow[t]{2}{*}{ Sí } & \% situación laboral & $18,2 \%$ & $16,8 \%$ & $12,7 \%$ & \multirow[t]{2}{*}{$16,1 \%$} \\
\hline & & Residuos corregidos & 1,3 & 0,4 & $-1,8$ & \\
\hline & \multirow[t]{2}{*}{ No } & \% situación laboral & $81,8 \%$ & $83,2 \%$ & $87,3 \%$ & \multirow[t]{2}{*}{$83,9 \%$} \\
\hline & & Residuos corregidos & $-1,3$ & $-0,4$ & 1,8 & \\
\hline Total & \multicolumn{2}{|c|}{ \% situación laboral } & $100 \%$ & $100 \%$ & $100 \%$ & $100 \%$ \\
\hline
\end{tabular}

En esta ocasión, todos los índices asociados (tanto Chi-cuadrado9 como los residuos corregidos) expresan la ausencia de relación entre ambas variables: situación laboral y violencia tipo A. Es decir, la probabilidad de ser víctima de violencia es independiente de la actividad laboral de las mujeres.

A través de esta misma encuesta, se pudo comprobar, igualmente, la ausencia de relación entre variables como la valoración de la opinión de la encuestada respecto a temas importantes que afectan al hogar por parte de la pareja y su situación laboral; o entre esta valoración y el hecho de ser la mujer la principal aportadora de ingresos al hogar. Es más, con un análisis más exhaustivo, a través de tablas de contingencia y estudio de residuos corregidos (Espinar, 2004: 259-260), únicamente puede hablarse de una cierta relación en la medida en que es más probable que, siendo ama de casa en exclusividad, estas opiniones sean más valoradas. Igualmente, las opiniones de las mujeres parecen ser valoradas en mayor medida cuando éstas no son las principales aportadoras de

9. Con un valor de 3,582; 2 grados de libertad y una significación asintótica de 0,0167. 
ingresos en la familia. En definitiva, cuando las familias se aproximan a los roles tradicionales, parecen ser más valoradas las opiniones de las mujeres (en ningún momento podemos olvidar el papel que en estos resultados puedan desempeñar los diferentes grados de conciencia de las mujeres hacia la desvalorización de sus opiniones, que podría variar a raíz de su participación en el mundo laboral).

Resumiendo, la participación laboral de las mujeres, con todo lo que ello supone, no parece implicar, necesariamente, cambios radicales en las relaciones de poder en el interior de las familias, en los roles de hombres y mujeres, en la propia identidad o en términos de violencia directa.

\section{Conclusiones}

Entre todos los términos disponibles en el campo de estudio que nos ocupa, hemos optado por emplear el de violencia de género, a raíz de sus potencialidades analíticas y prácticas.

Analíticamente, el término violencia de género incluye, en su misma denominación, cuáles son las raíces de la violencia a la que hace referencia: las definiciones y relaciones de género dominantes en una sociedad dada. De esta forma, permite analizar, conjuntamente, diferentes formas de violencia; todas ellas basadas, en última instancia (e independientemente de la intervención de otras variables), en unos mismos fundamentos socioculturales: unas relaciones de poder desiguales entre hombres y mujeres y unos prejuicios y una creencias desvalorizadoras de lo femenino.

En términos prácticos, hablar de violencia de género supone destacar el fundamento sociocultural de esta violencia, abriendo la posibilidad de cambios e indicando las áreas sobre las que actuar. Es más, la violencia de género queda definida como un problema de carácter social y no, meramente, individual. Es decir, por sus dimensiones estructurales y culturales, se trata de un problema cuyas causas, consecuencias y, por tanto, medidas, implican al conjunto de la sociedad y no únicamente a los individuos directamente afectados.

De acuerdo con estos planteamientos, resulta perfectamente aplicable a la violencia de género el triángulo de la violencia que en su día definiera Johan Galtung. Este autor plantea la necesidad de diferenciar tres formas de violencia: directa, estructural y cultural. Así, la violencia estructural y la cultural, que, en sí mismas, pueden considerarse violencia dados sus efectos sobre quienes las padecen, pueden también interpretarse como el fundamento de la violencia directa.

Igualmente, si el objetivo es poner fin a esta violencia, no es suficiente (aunque irrenunciable) actuar sobre las manifestaciones de violencia directa, sino que sería necesario atender a las otras dos formas de violencia: la estructural y la cultural.

En este sentido, hemos analizado los posibles efectos derivados de un cambio estructural como es la reincorporación de las mujeres al mercado laboral sobre las otras dos dimensiones de la violencia: la cultural y la directa. Así, 
observando sus características, hemos podido constatar las claras debilidades de este cambio estructural: mantenimiento del reparto tradicional de responsabilidades en el interior de los hogares, segmentación horizontal y mayor precariedad laboral en el caso de las mujeres (paro, contratos temporales y a tiempo parcial, trabajo informal). En definitiva, podemos calificar esta transformación de insuficiente, llegando a cuestionar los posibles efectos que pueda tener en términos de cambios en las definiciones de roles e identidades, $y$, en general, en términos de violencia cultural y directa. Es más, podemos concluir que la persistencia de unas determinadas creencias en torno a las funciones, a los roles y a las capacidades de hombres y mujeres están configurando las características que, en general, presenta la participación laboral de las mujeres.

Desde otro nivel de análisis, hemos podido comprobar, a través de datos de encuesta, que tampoco parece posible establecer una relación estadísticamente significativa entre la participación laboral de las mujeres y una menor probabilidad, para las mismas, de sufrir violencia directa; en todo caso, es posible hablar, incluso, de la existencia de relación en el sentido contrario.

Partiendo de estas observaciones, podemos concluir, tal y como adelantábamos anteriormente, que, si el objetivo es poner fin a la violencia de género, la acción ha de dirigirse a los tres elementos que la componen: la violencia directa, estructural y cultural. Así, será necesario reforzar los cambios estructurales iniciados, superando sus debilidades; a la vez que se hace inevitable una profunda transformación del ámbito cultural que, de otra forma, continuará justificando el mantenimiento de relaciones de dominación y de violencia.

\section{Bibliografía}

Alberdi, I. (1999). «El significado del género en las ciencias sociales». Política y Sociedad, 32, p. 9-21.

Beauvoir, S. (1949). El segundo sexo, vol. I y II. Madrid: Cátedra, Universitat de València, Instituto de la Mujer, 2000.

BONINO, L. (2003). «Los varones ante el problema de la igualdad con las mujeres». En: LOMAS, C. (ed.) ¿Todos los hombres son iguales?: Identidad masculina y cambios sociales. Barcelona: Paidós.

BREINES, I.; CONNELL, R.; EIDE, I. (eds.) (2000). Male roles, masculinities and violence. A culture of peace perspective. París: UNESCO.

Brod, H.; KaUfMan, M. (eds.) (1994). Theorizing Masculinities. Thousand Oaks, California: Sage.

BUTLER, J. (1990). Gender trouble. Feminism and the subversion of identity. Nueva York y Londres: Routledge.

CAMPBeLl, J. (1993). «Prevention of wife battering; Insights from cultural análisis». Response, 80, p. 18-24.

Carrasco, C.; MaYordomo, M. (2000). «Los modelos y estadísticas de empleo como construcción social: la encuesta de población activa y el sesgo de género». Politica y Sociedad, 34, p. 101-112. 
Carrasco, C.; Mayordomo, M.; Domínguez, M.; Alabart, A. (2004). Trabajo con mirada de mujer: Propuesta de una encuesta de población activa no androcéntrica. Madrid: CES.

CASTAÑO, C. (dir.) (2004). Indicadores laborales básicos de la situación de la mujer en España y sus regiones. Madrid: Instituto de la Mujer.

Cово, R. (1995). «Género». En: Amorós, Celia (dir.). 10 palabras clave sobre mujer. Estella: Verbo Divino, p. 55-84.

Connell, R. W. (1995). Masculinities. University of California Press.

CORSI, J.; BONINO, L. (2003). «Violencia y género: la construcción de la masculinidad como factor de riesgo». En: CORSI, Jorge; PeYrú, Graciela M. (coords.). Violencias sociales. Barcelona: Ariel, p. 117-138.

Dutton, D. G.; Kropp, P. R. (2000). «A Review of Domestic Violence Risk Instruments». En: Trauma, violence and abuse, vol. 1, n' 2 , p. 171-181.

ESPINAR, E. (2004). Violencia de género y procesos de empobrecimiento: Estudio de la violencia contra las mujeres por parte de su pareja o ex-pareja sentimental. Alicante: Biblioteca Virtual Miguel de Cervantes.

Felson, R. B. (2002). Violence and Gender Reexamined. Washington, D. C.: American Psychological Association.

Follingstad, D. R.; Bradley, R. G.; HelfF, C. M.; Laughlin, J. E. (2002). «A model for predicting dating violence: Anxious attachment, angry temperament and need for relationship control». Violence and Victims, 17 (91), p. 35-47.

Gabler, M.; STern, S. E.; Miserandino, M. (1998). «Latin American, Asian, and American cultural differences in perceptions of spousal abuse». Psychological Reports, 83, p. 587-592.

Galtung, J. (1990). "Cultural Violence». Journal of Peace Research, 27, $\mathrm{n}^{\circ} 3$, p. 291-305.

GALTUNG, J. (1995). Investigaciones teóricas. Sociedad y cultura contemporáneas. Madrid: Tecnos / Instituto de Cultura Juan Gil-Albert.

Galtung, J. (1996). Peace by peaceful means. Peace and conflict, development and civilization. Oslo: International Peace Research Institute (PRIO).

Heise, L.; EllsberG, M.; GotTMOeller, M. (1999). Ending violence against women. Populations Reports. Baltimore: Johns Hopkins University.

HirigoYen, M. F. (1998). Le Harcèlement moral: la violence perverse au quotidien. París: Éditions La Découverte \& Syros.

HoltzWorth-Munroe, A.; Bates, L.; SMutzler, N.; Sandin, E. (1997). «A brief review of the research on husband violence». Aggression and Violent Behavior, 1, p. 65-99.

INSTITUTO DE LA MUJER (2000). La violencia contra las mujeres. Resultados de la macroencuesta. Madrid: Ministerio de Trabajo y Asuntos Sociales. Instituto de la Mujer.

IZQUIERDO, M. J. (1998). «Los órdenes de la violencia: especie, sexo y género». En: FISAS, V. (ed.). El sexo de la violencia. Género y cultura de la violencia. Barcelona: Icaria, p. 61-91.

KAUFMAN, M. (1999). «La construcción de la masculinidad y la triada de la violencia masculina». En: VV. AA. Violencia doméstica. Cuernavaca-Morelos (México). Cidhal, PRODEC, Centro de Documentación Betsie Hollants, p. 52-69.

Lipovetsky, G. (1997). La tercera mujer. Permanencia y revolución de lo femenino. Barcelona: Anagrama. (1999).

LÓPEZ, I.; SIERRA, B. (2001). Integrando el análisis de género en el desarrollo. Manual para técnicos de cooperación. Madrid: IUDC/UCM. 
LÓPEZ, M. T.; VAliÑo, A. (2003). «El trabajo asalariado como generador de derechos sociales. Las prestaciones económicas del sistema de Seguridad Social: diferencias entre hombres y mujeres». En: SARASÚA, C.; GÁLVEZ, L. (eds.). ¿Privilegios o eficiencia? Mujeres y hombres en los mercados de trabajo. Alicante: Publicaciones de la Universidad de Alicante, p. 381-401.

MAGAllón, C. (2005). «Epistemología y violencia. Aproximación a una visión integral sobre la violencia hacia las mujeres». Feminismo/s, 6, p. 33-47.

MARKOWITZ, F. E. (2001). «Attitudes and family violence: Linking intergenerational and cultural theories». Journal of Family Violence, 16, p. 205-218.

MARTín, E.; MARTíN, M. (1999). Las violencias cotidianas cuando las víctimas son las mujeres. Madrid: Ministerio de Trabajo y Asuntos Sociales. Instituto de la Mujer.

MARTíneZ, M. A. (2005). "Violencias estructurales: obstáculos para el cumplimiento de los derechos humanos de las mujeres pobres». Feminismo/s, 6, p. 49-64.

MARUANI, M. (2004). «Hombres y mujeres en el mercado de trabajo: paridad sin igualdad». Revista de Economía Mundial, no 10/11, p. 59-75.

MeIL, G. (2004). «Cambio familiar y maltrato conyugal a la mujer». RIS, no 37. p. 7-27.

MiedZIAN, M. (1991). Boys will be boys: Breaking the link between masculinity and violence. Nueva York: Anchor / Doubleday.

Nayak, M. B.; Byrne, C. A.; Martin, M. K.; Abraham, A. G. (2003). «Attitudes Toward Violence Against Women: A Cross-Nation Study». Sex Roles, 49 (7/8), p. 333-342.

PRIETO, C (1999). «Los estudios sobre mujer, trabajo y empleo: caminos recorridos, caminos por recorrer». Politica y Sociedad, 32, p. 141-149.

PRINGLE, K. y otros (2001). "The European Research Network on Men in Europe: The Social Problem of Men». Journal of European Social Policy, vol. 11, $\mathrm{n}^{\circ}$ 2, p. 171-173.

RALDÚA, E. V. (2001). «Comparación internacional de los empleos del tiempo de mujeres y hombres». REIS, no 94, p. 105-126.

ReVerter, S. (2003). «La perspectiva de género en la filosofía». Feminismo/s, 1, p. 3350.

RiVERA, M. M. (2001). «La violencia contra las mujeres no es violencia de género». Duoda. Revista d'Estudis Feministes, $\mathrm{n}^{\circ} 21$.

SAU, V. (2000). Diccionario ideológico feminista, I. Barcelona: Icaria.

TORres, M. (2001). La violencia en casa. México: Paidós.

TorTosa, J. M. (1992). Sociología del sistema mundial. Madrid: Tecnos.

Tortosa, J. M. (coord.) (2001). Pobreza y perspectiva de género. Barcelona: Icaria.

Tortosa, J. M. (2002). "Recomendaciones para el estudio de las violencias». Alternativas, $\mathrm{n}^{\circ} 10$, p. 19-36.

TORTOSA, J. M. (coord.) (2002). Mujeres pobres, indicadores de empobrecimiento en la España de hoy. Madrid: Cáritas, Fundación Foessa

TuberT, S. (ed.) (2003). Del sexo al género. Los equívocos de un concepto. Madrid: Cátedra, Universitat de València, Instituto de la Mujer. 